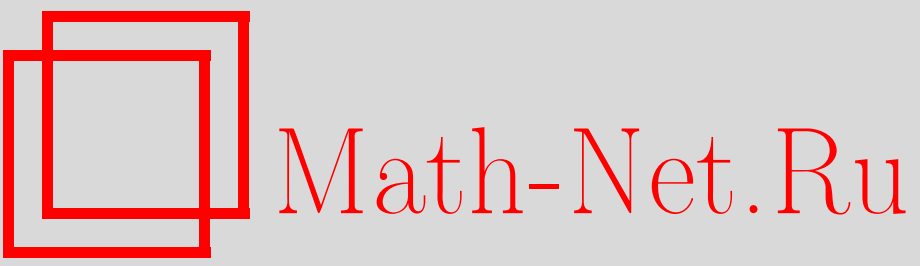

А. А. Арсеньев, Оценка мнимой части полюса матрицы рассеяния для трехмерного уравнения Шредингера с ловушечным потенциалом, ТМФ, 1998, том 114, номер 2, 271-276

DOI: https://doi.org/10.4213/tmf839

Использование Общероссийского математического портала Math-Net.Ru подразумевает, что вы прочитали и согласны с пользовательским соглашением

http: //www . mathnet.ru/rus/agreement

Параметры загрузки:

IP : 54.196 .121 .252

26 апреля 2023 г., 15:15:13 
(C) 1998 г.

\section{ОЦЕНКА МНИМОЙ ЧАСТИ ПОЛЮСА МАТРИЦЫ РАССЕЯНИЯ ДЛЯ ТРЕХМЕРНОГО УРАВНЕНИЯ ШРЕДИНГЕРА С ЛОВУШЕЧНЫМ ПОТЕНЦИАЛОМ}

\footnotetext{
Предложен метод оценки мнимой части резонансного полюса матрицы рассеяния для трехмерного уравнения Шредингера с ловушечным потенциалом. Метод основан на принципе инвариантности волновых операторов и равенстве Парсеваля. Показано, что при росте высоты барьера мнимая часть резонансного полюса матрицы рассеяния стремится к нулю экспоненциально.
}

Действительная неотрицательная функция $V: R^{3} \rightarrow R_{+}^{1}$ называется ловушечным потенциалом, если она принимает большие значения в слое $\{x ; a<|x|<b\}$, а ее значения вне этого слоя малы (математическое определение будет дано ниже). Хорошо известно [1], что аналитическое продолжение матрицы рассеяния для уравнения Шредингера с ловушечным потенциалом имеет полюсы с малыми мнимыми частями (на этом, по сушеству, основана классическая математическая модель гамовской теории $\alpha$-распада: период полураспада отождествляется с обратной величиной мнимой части полюса матрицы рассеяния). Методам оценки мнимой части полюса матрищы рассеяния посвящено большое количество работ [2-6]. Эти методы в большинстве своем основаны на следуюшем приеме: резольвента оператора Шредингера рассматривается как интегральный оператор во вспомогательном банаховом пространстве и ишутся полюсы этого оператора. Для аналитических потенциалов этот метод иногда используется в комбинации с методом комплексного скейлинга, в сферически-симметричном случае удается использовать аналитическую теорию обыкновенных дифференциальных уравнений. Однако окончательньй результат редко удается получить в обозримой и физически осмысленной форме. С математической точки зрения трудной является проблема ложных полюсов, поэтому задача оценки мнимой части резонансных полюсов матрицы рассеяния продолжает привлекать внимание исследователей. В данной работе мы указываем на возможность использования в этой задаче принципа инвариантности волновых операторов Бирмана и равенства Парсеваля для собственныхх функций непрерывного спектра оператора Шредингера. Насколько нам известно, ранее они в подобной ситуации не использовались. Мы дадим оценку мнимой части резонансных полюсов матрицы рассея-

\footnotetext{
* Московский государственный университет, Москва, Россия
} 
ния через параметры потенциала в "евклидовой области". Основной результат работы изложен в теоремах 1 и 2.

Исходным пунктом наших рассуждений является предложенная в [7] модель резонансного рассеяния на ловушечном потенциале. Напомним ее.

Пусть $V_{\infty}(x)$ - неотрицательная функция: $V_{\infty}: R^{3} \rightarrow R_{+}^{1}$, которая принимает значения $+\infty$ на ограниченном множестве $\Omega=\left\{x ; V_{\infty}(x)=+\infty\right\}$, дополнение $C \Omega$ которого состоит из непустого ограниченного множества $D_{\mathrm{int}}$ и содержит внешность шара связного множества $D_{\text {out }}$,

$$
C \Omega=D_{\text {int }} \cup D_{\text {out }}, \quad \operatorname{mes} D_{\text {int }}>0, \quad \operatorname{dist}\left(D_{\text {int }}, D_{\text {out }}\right)>0 .
$$

Предположим, что при любом $n<\infty$ функция $\min \left(n, V_{\infty}(x)\right)$ локально удовлетворяет условию Гельдера и вне некоторого шара верна оценка

$$
V_{\infty}(x)<\text { const } \exp \left(-a|x|^{2}\right), \quad a>0, \quad|x|>R_{0} .
$$

Пусть $V_{n}(x)$ - монотонно возрастаюшая последовательность непрерывных по Гельдеру функций, для которой

$$
\min \left(n, V_{\infty}(x)\right) \leq V_{n}(x) \leq \min \left(n+1, V_{\infty}(x)\right) .
$$

Функцию $V_{n}(x)$ при достаточно большом $n$ мы называем ловушечным потенциалом, символом $H_{n}$ обозначаем оператор Шредингера с потенциалом $V_{n}(x)$ :

$$
H_{n}=-\nabla^{2}+V_{n} .
$$

При $n=\infty$ по определению полагаем

$$
H_{\infty}=-\left.\partial_{t}\left(\lim _{n \rightarrow \infty} \exp \left(-t H_{n}\right)\right)\right|_{t=0} .
$$

В [7] доказано, что определенный формулой (1) оператор самосопряжен в $L^{2}(C \Omega, d x)$. Решением задачи рассеяния мы называем функцию $u_{n}(x, \omega, \lambda)$, которая удовлетворяет уравнению $H_{n} u_{n}=\lambda u_{n}, \lambda>0$, и представима в виде

$$
\begin{gathered}
u_{n}(x, \omega, \lambda)=\exp (i(x, \omega) \sqrt{\lambda})+\varphi_{n}(x, \omega, \lambda), \quad|\omega|=1, \\
\left(\partial_{x}-i \sqrt{\lambda}\right) \varphi_{n}(x, \omega, \lambda)=o\left(|x|^{-1}\right), \quad \varphi_{n}(x, \omega, \lambda)=O\left(|x|^{-1}\right), \quad|x| \rightarrow \infty,
\end{gathered}
$$

где $(,$,$) - скалярное произведение в R^{3}$. При $n<\infty$ функции $u_{n}(x, \omega, \lambda)$ образуют полную в $L^{2}\left(R^{3}, d x\right)$ систему собственных функций оператора $H_{n}$. Равенство Парсеваля имеет вид

$$
\int|f(x)|^{2} d x=(2 \pi)^{-3}(1 / 2) \int_{0}^{\infty}\left[\int_{S}\left|\left\langle f \mid u_{n}\right\rangle(\omega, \lambda)\right|^{2} d \omega\right] \lambda^{1 / 2} d \lambda,
$$


где

$$
\left\langle f \mid u_{n}\right\rangle(\omega, \lambda)=\int f(x) u_{n}(x, \omega, \lambda) d x,
$$

интегрирование без обозначения пределов проводится по всему пространству, $S$ - единичная сфера в $R^{3}, d \omega$ - элемент поверхности единичной сферы.

Как доказано в [7], сужение оператора $H_{\infty}$ на $L^{2}\left(D_{\mathrm{int}}, d x\right)$ имеет дискретный спектр.

Пусть $\psi\left(x, \lambda_{j}\right)$ - продолженная нулем на $C D_{\text {int }}$ простая собственная функция дискретного спектра оператора $H_{\infty}$, которая отвечает собственному значению $\lambda_{j}$. Положим

$$
a_{j}(\lambda, n)=\int_{S}\left|\left\langle\psi\left(\cdot, \lambda_{j}\right) \mid u_{n}\right\rangle(\lambda, \omega)\right|^{2} d \omega
$$

Следуюшая лемма является переформулировкой утверждений лемм 10 и 22 работы [7] в удобных для нас обозначениях, и мы не будем ее доказывать.

ЛЕмма 1. Пусть по достаточно велико и в достаточно мало. Тогда при $n_{0}<$ $n<\infty,\left|\lambda-\lambda_{j}\right|<\varepsilon$ определенная формулой (2) функиия представима в виде

$$
a_{j}(\lambda, n)=b(\lambda, n)\left|\operatorname{Im} \mu_{n}(\lambda)\right|\left|1-\mu_{n}(\lambda)\right|^{-2}+c(\lambda, n)
$$

где $b, c, \mu_{n}$ - аналитические в окрестности $\left\{\lambda ;\left|\lambda-\lambda_{j}\right|<\varepsilon\right\}$ функиии, которые равномерно по $\lambda$ имеют предел при $n \rightarrow \infty$ и обладают следующими свойствами:

$$
b(\lambda, n)>\text { const }>0, \quad|c(\lambda, n)|<\text { const }<\infty, \quad\left|\partial \mu_{n} / \partial \lambda\right|>\text { const }>0 .
$$

Уравнение $1-\mu_{n}(\lambda)=0$ имеет единственный корень $\lambda_{j, n}$, который является полюсом матрицы рассеяния и таков, что $\operatorname{Im} \lambda_{j, n}<0, \lambda_{j, n} \rightarrow \lambda_{j}, \quad n \rightarrow \infty$.

Символом const мы обозначаем положительную, не зависящую от параметра $n$ величину, значения которой зависят от каждого вхождения символа в формулу. Под матрицей рассеяния мы понимаем стандартно определенную по [8] матрицу и рассматриваем ее как аналитическую функцию параметра $\lambda$ со значениями в пространстве операторов над $L^{2}(S, d \omega)$.

Положим

$$
G_{n}(t)=\exp \left(-t H_{n}\right), \quad t>0 .
$$

Пусть $G_{n}(x, y, t)$ - интегральное ядро оператора $G_{n}(t)$.

Лемма 2. При $n<\infty$ справедливо равенство

$$
\begin{gathered}
(2 \pi)^{-3}(1 / 2) \int_{0}^{\infty}\left[\int_{S}\left|\exp \left(-\lambda_{j} t\right)-\exp (-\lambda t)\right|^{2}\left|\left\langle\psi\left(\cdot, \lambda_{j}\right) \mid u_{n}\right\rangle(\omega, \lambda)\right|^{2} d \omega\right] \lambda^{1 / 2} d \lambda= \\
=\left\|\left(G_{n}(t)-G_{\infty}(t)\right) \psi\left(\cdot, \lambda_{j}\right)\right\|^{2}
\end{gathered}
$$

где норма берется в пространстве $L^{2}\left(R^{3}, d x\right)$ 
ДОКАЗАТЕЛЬСтво. Справедливы равенства

$$
\begin{aligned}
\exp \left(-\lambda_{j} t\right) \psi\left(x, \lambda_{j}\right) & =\int G_{\infty}(x, y, t) \psi\left(y, \lambda_{j}\right) d y \\
\exp (-\lambda t) u_{n}(x, \omega, \lambda) & =\int G_{n}(x, y, t) u_{n}(y, \omega, \lambda) d y
\end{aligned}
$$

откуда следует, что

$$
\left(\exp (-\lambda t)-\exp \left(-\lambda_{j} t\right)\right)\left\langle\psi\left(\cdot, \lambda_{j}\right) \mid u_{n}\right\rangle(\omega, \lambda)=\int \varphi(y) u_{n}(y, \omega, \lambda) d y
$$

где

$$
\varphi(y)=\int\left(G_{n}(y, x, t)-G_{\infty}(y, x, t)\right) \psi\left(x, \lambda_{j}\right) d x .
$$

Следовательно, (3) есть равенство Парсеваля для функции $\varphi$. Лемма доказана.

Положим

$$
\begin{gathered}
m(\sigma)=\left\{\lambda ;\left|\lambda-\lambda_{j}\right|>\sigma\right\}, \quad d(\sigma)=\left\{\lambda ;\left|\lambda-\lambda_{j}\right|<\sigma\right\}, \\
I(\sigma, n)=(2 \pi)^{-3}(1 / 2) \int_{m(\sigma)} a_{j}(\lambda, n) \lambda^{1 / 2} d \lambda, \\
\theta(t, n)=\left\|G_{n}(t)-G_{\infty}(t)\right\|,
\end{gathered}
$$

где берется операторная норма в $L^{2}\left(R^{3}, d x\right)$.

ЛЕмма 3. При $t>0, n<\infty$ справедливо неравенство

$$
I(\sigma, n) \leq \exp \left(2 \lambda_{j} t\right)(1-\exp (-\sigma t))^{-2} \theta(t, n)^{2} .
$$

ДоКАЗАТЕЛЬСТво. Из (3) следует, что

$$
\theta(t, n)^{2} \geq\left\|\left(G_{n}(t)-G_{\infty}(t)\right) \psi\left(\cdot, \lambda_{j}\right)\right\|^{2} \geq \inf _{\lambda \in m(\sigma)}\left|\left(\exp \left(-\lambda_{j} t\right)-\exp (-\lambda t)\right)\right|^{2} I(\sigma, n) .
$$

Лемма доказана.

Теорема 1. Для находящихся в окрестности $\left\{\lambda ;\left|\lambda-\lambda_{j}\right|<\varepsilon\right\}$ полюсов матрицы рассеяния справедлива оценка

$$
\left|\operatorname{Im} \lambda_{j, n}\right|<\operatorname{const} \theta(t, n)
$$

где $t>0$ - произвольньй параметр, а константа зависит от параметра $t$ и потенциала $V_{\infty}(x)$. 
ДокАЗАТЕЛЬСТво. Пусть $\sigma(n)$ - произвольная последовательность, удовлетворяюшая условию

$$
\sigma(n) \rightarrow 0, \quad \theta(t, n) / \sigma(n) \rightarrow 0, \quad n \rightarrow \infty
$$

Воспользовавшись равенством Парсеваля для функции $\psi\left(x, \lambda_{j}\right)$, получим

$$
\begin{aligned}
1 & =(2 \pi)^{-3}(1 / 2) \int_{d(\sigma(n))} a_{j}(\lambda, n) \lambda^{1 / 2} d \lambda+I(\sigma(n), n)= \\
& =\int_{d(\sigma(n))} b(\lambda, n)\left|\operatorname{Im} \mu_{n}(\lambda)\right|\left|1-\mu_{n}(\lambda)\right|^{-2} \lambda^{1 / 2} d \lambda+o(1)< \\
& <\operatorname{const} \int_{d(\sigma(n))}\left|1-\mu_{n}(\lambda)\right|^{-1} d \lambda+o(1)< \\
& <\operatorname{const} \sigma(n) /\left|\operatorname{Im} \lambda_{j, n}\right|+o(1), \quad n \rightarrow \infty .
\end{aligned}
$$

В приведенных выше выкладках мы сначала учли лемму 1 и неравенство (4), потом воспользовались неравенством

$$
\left|\operatorname{Im} \mu_{n}(\lambda)\right|=\mid \operatorname{Im}\left(1-\mu_{n}(\lambda)|\leq| 1-\mu_{n}(\lambda) \mid .\right.
$$

Затем мы учли, что в силу леммы 1

$$
\left|1-\mu_{n}(\lambda)\right|=\left|\mu_{n}\left(\lambda_{j, n}\right)-\mu_{n}(\lambda)\right| \geq \mathrm{const}\left|\lambda_{j, n}-\lambda\right| \geq \mathrm{const}\left|\operatorname{Im} \lambda_{j, n}\right| .
$$

Из (7) следует, что

$$
\left|\operatorname{Im} \lambda_{j, n}\right|<\operatorname{const} \sigma(n) .
$$

Так как (8) выполнено для любой удовлетворяющей условию (6) последовательности, должно быть выполнено неравенство (5). Теорема доказана.

Положим

$$
\beta_{n}(x, t)=\int\left(G_{n}(x, y, t)-G_{\infty}(x, y, t)\right) d y .
$$

Из известной оценки Карлемана для нормы интегрального оператора следует, что

$$
\theta(t, n) \leq \sup _{x} \beta_{n}(x, t)
$$

Простейшую оценку функции $\beta_{n}(x, t)$ можно получить на основе представления решения задачи Коши для уравнения теплопроводности через математическое ожидание от функционала броуновской траектории:

$$
\int G_{n}(x, y, t) d y=E\left(\exp \left(-t \int_{0}^{1} V_{n}(2 \sqrt{t} x(\tau)+x) d \tau\right)\right) .
$$

Здесь броуновское движение нормировано так, что $E\left(x_{i}(\tau) x_{j}(\tau)\right)=2 \delta_{i j} \tau$. Пусть

$$
\begin{aligned}
V_{n}(x)= & \min \left(n, V_{\infty}(x)\right), \\
\delta(n)= & \min \left(\operatorname{dist}\left(\left\{x ; V_{n}(x)<n / 4\right\},\left\{x ; V_{n}(x)>n / 2\right\}\right),\right. \\
& \left.\operatorname{dist}\left(\left\{x ; V_{n}(x)<n / 2\right\},\left\{x ; V_{n}(x)=n\right\}\right)\right) .
\end{aligned}
$$


ТЕОрема 2. Для входящей в оценку (5) величинь $\theta(t, n)$ справедлива оченка (11).

ДокАЗАТЕЛЬСТво. В области $\left\{x ; V_{n}(x)>n / 2\right\}$ из (10) следует, что

$$
\begin{aligned}
\beta_{n}(x, t) & \leq \exp \left(-t \min _{|\zeta|<\delta(n)} V_{n}(x+\zeta)\right)+E\left(\sup _{\tau}|x(\tau)|>\delta(n) / 2 \sqrt{t}\right) \leq \\
& \leq \exp (-t n / 4)+2 \sqrt{2} \exp \left(-\delta(n)^{2} / 32 t\right) .
\end{aligned}
$$

В области $\left\{x ; V_{n}(x)<n / 2\right\}$ мы имеем

$$
\begin{aligned}
\beta_{n}(x, t) & \leq E\left(1-\exp \left(-t \int_{0}^{1}\left(V_{\infty}-V_{n}\right)(2 \sqrt{t} x(\tau)+x) d \tau\right)\right) \leq \\
& \leq E\left(\sup _{\tau}|x(\tau)|>\delta(n) / 2 \sqrt{t}\right)<2 \sqrt{2} \exp \left(-\delta(n)^{2} / 32 t\right),
\end{aligned}
$$

следовательно,

$$
\sup _{x} \beta_{n}\left(x, t_{0}\right)<4 \sqrt{2} \exp (-\delta(n) \sqrt{n} / 8 \sqrt{2}), \quad t_{0}=\delta(n) /(2 \sqrt{2 n}) .
$$

Однако для любого целого $m$

$$
\theta(t, n) \leq m \theta(t / m, n) \leq m \sup _{x} \beta_{n}(x, t / m),
$$

и поэтому

$$
\theta(t, n) \leq 4 \sqrt{2}(1+t 2 \sqrt{2 n} / \delta(n)) \exp (-\delta(n) \sqrt{n} / 8 \sqrt{2})
$$

Теорема доказана.

Если, например,

$$
V_{\infty}(x) \cong(\operatorname{dist}(x, \partial \Omega))^{-q}, \quad q>2,
$$

то оценка (11) дает

$$
\left|\operatorname{Im} \lambda_{j, n}\right|<\text { const }_{1} \exp \left(- \text { const }_{2} n^{(1 / 2-1 / q)}\right),
$$

где положительные константы зависят только от $V_{\infty}$.

Работа выполнена при поддержке Российского фонда фундаментальных исследований, проект № 96-01-00084.

\section{Список литературы}

[1] А.И. Базь, Я.Б. Зельдович, А. М. Переломов. Рассеяние, реакции и распады в нерелятивистской квантовой механике. М.: Наука, 1966.

[2] Resonances: The unifying route towards the formulation of dynamical processes. Eds E. Brandas, N. Elander. Lecture Notes in Physics. V. 325. Berlin-Heidelberg-New York: Springer-Verlag, 1987.

[3] M.S. Asbaugh, E. M. Harrell. Commun. Math. Phys. 1982. V. 83. P. 151-170.

[4] E. Enghal, E. Brandas. Phys. Rev. A. 1988. V. 37. P. 4145-4152.

[5] S. Albeverio, R. Hoegh-Kron. J. Math. Anal. and Appl. 1984. V. 101. P. 491-513.

[6] W. Hunziker. Commun. Math. Phys. 1990. V. 132. P. 177-188.

[7] А. А. Арсеньев. ТМФ. 1995. Т. 104. № 2. С. 214-232.

[8] М. ШІ. Бирман, С. Б. Энтина. Изв. АН СССР. Сер. матем. 1967. Т. 31. С. 401-430. 